Relations industrielles

Industrial Relations

\title{
Le cinquième Congrès de l'A.P.I.
}

\section{Gaston Cholette}

Volume 5, numéro 3, décembre 1949

URI : https://id.erudit.org/iderudit/1023295ar

DOI : https://doi.org/10.7202/1023295ar

Aller au sommaire du numéro

\section{Éditeur(s)}

Département des relations industrielles de l’Université Laval

\section{ISSN}

0034-379X (imprimé)

1703-8138 (numérique)

Découvrir la revue

Citer cet article

Cholette, G. (1949). Le cinquième Congrès de l'A.P.I. Relations industrielles /

Industrial Relations, 5(3), 26-28. https://doi.org/10.7202/1023295ar

Tous droits réservés @ Département des relations industrielles de l’Université Laval, 1949
Ce document est protégé par la loi sur le droit d'auteur. L’utilisation des services d'Érudit (y compris la reproduction) est assujettie à sa politique d'utilisation que vous pouvez consulter en ligne.

https://apropos.erudit.org/fr/usagers/politique-dutilisation/ 
Il était clair pour la plupart des personnes présentes que le congrès avait fait beaucoup pour atteindre le but qu'il s'était fixé. Beaucoup de points d'interrogation ont disparu et beaucoup d'incompréhensions ont été dissipées au cours de la discussion, mais, à la longue, on finira peut-être par se rendre compte que le plus grand succès remporté par ce congrès, c'est le fait même qu'il ait été organisé. Le seul fait de tenir des réunions de ce genre était une franche admission de la tension qui existait et de la nécessité de se parler franchement pour se comprendre de part et d'autre, si l'on veut que règnent la paix et la prospérité. C'est probablement là la raison pour laquelle tous ceux qui m'ont parlé au terme de ces assi- ses ont été unanimes à rendre hommage à la clairvoyance et au courage dont a fait preuve M. l'abbé Gérard Dion en organisant et en dirigeant ce congrès.

D'autres réunions semblables dans l'avenir auront sans doute beaucoup de succès, car le précédent qui a été créé comportait une méthode, une atmosphère et des objectifs qui forment une base solide pour l'édification de nouvelles initiatives du même genre. Pour quelqu'un des Etats-Unis qui voit les choses du dehors, il apparaît clairement que ces deux jours de novembre à l'hôtel Kent House ont rassemblé trop de gens de bonne volonté et ont été trop gorgés de lumière pour ne pas être le prélude d'heureux lendemains.

\section{LE CINQUIÈME CONGRÈS DE L'A.P.I.}

Gaston Cholette

L'Association professionnelle des industriels a tenu un brillant congrès à Québec, les 14 et 15 novembre. La mise en scène était excellente et le prestige autant que la valeur des conférenciers donnait beaucoup d'éclat à ces assises patronales.

Les organisateurs avaient jugé opportun d'épingler au discours du 7 mai de Pie XII, dont le texte faisait l'objet des études des congressistes, le thème suivant qui lui servait d'étiquette: «Le patron et son entreprise ».

La première conférence, portant sur la collaboration patronale-ouvrière, fut donnée par $\mathbf{M}$. Gilbert Ayers, président de la Compagnie Ayers Limited de Lachute. On fit ressortir, au cours de cet exposé et de la discussion qui suivit, l'importance de mesures avancées de sécurité sociale d'initiative privée, leur coût relativement peut élevé et leurs répercussions heureuses sur les ouvriers, surtout au moment des demandes d'augmentation de salaires.

Après le déjeûner, au cours de la première journée, une solide pièce de philosophie sur le droit de propriété fut présentée par un universitaire de grande renommée, le Père Louis Lachance, o. p. Tel qu'il convenait, le conférencier mit en lumière les fondements traditionnels du droit de propriété en général, les éléments justificatifs de la propriété privée et, surtout, la fonction sociale de celle-ci. La satisfaction universelle des besoins humains est une des exigences primaires de la loi naturelle, et la propriété privée n'est valable que dans la mesure où elle constitue un moyen efficace de remplir ce rôle. Après le déjeûner, le soussigné a demandé au Père Lachance si l'on pouvait interpréter certaines parties de son exposé comme l'expression d'une divergence d'opinion avec quelques conclusions de la brochure de la Commission sacerdotale d'études sociales sur «La participation des travailleurs à la vie de l'entreprise». Le savant dominicain répondit qu'il avait lu très attentivement cette publication et que rien, dans sa propre conférence, ne venait en contradiction avec la pensée de la Commission sacerdotale.

L'après-midi du lundi fut marquée par deux conférences très intéressantes: l'une sur l'organisation professionnelle, l'autre sur les réformes de structure dans l'entreprise. La première fut donnée par $\mathbf{M}$. Marcel Clément et la seconde par $\mathbf{M}$. François-Albert Angers. La conclusion la plus importante qui en ressort et qui, d'ailleurs, a fait l'objet d'une déclaration conjointe des deux conférenciers, est celle qui propose à l'A.P.I. la formation de sections professionnelles en son sein avec mission d'instituer une collaboration organique, régulière et systématique avec les branches correspondantes du syndicalisme ouvrier, en vue de réaliser le plus efficacement possible la collaboration patronale-ouvrière telle qu'elle est demandée dans les encycliques et les autres textes pontificaux. Cette exhortation pressante, vue dans la perspective du discours du 7 mai de Pie XII et dans ses rapports avec les discours de M. Vittorio Vaccari et de Mgr Maurice Roy, constitue l'un des meilleurs voeux qui aurait pu être formulé 
à cette occasion par une association patronale d'inspiration catholique.

La première journée fut clôturée par une conférence de $M$. Vittorio Vaccari, secrétaire général de l'Association des employeurs catholiques de Belgique, et par une allocution de Son Exc. Mgr Maurice Roy, archevêque de Québec. M. Vaccari avait été invité à parler des directives sociales de Pie XII, et notamment à interpréter son discours du 7 mai aux patrons. Voici quelques extraits capitaux de son texte. «On doit l'interpréter (ce discours de Pie XII) dans le cadre des directives sociales que, depuis l'encyclique Rerum Novarum à nos jours, la papauté n'a jamais cessé de donner aux peuples de la terre... Il y a quelqu un qui a voulu injustement relever dans ce discours un changement d'orientation en regard des principes énoncés dans d'autres documents... Et il faut dire qu'il y a en certains milieux de patrons qui, se bornant à un examen superficiel et fragmentaire du discours, ont mutilé sa construction organique, de telle façon qu'ils l'ont interprété comme une exposition des droits naturels du patron plutôt que de ses devoirs également ». Un peu plus loin, M. Vaccari affirme: «L'entreprise privée paraît être le milieu le plus apte à développer le rapport de responsabilité commune, pour autant que celle-ci est le produit vivant de la libre initiative des individus et de leurs groupes librement constitués ».

Mgr Maurice Roy, archevêque de Québec, insista sur deux points particuliers: les patrons doivent prendre eux-mêmes l'initiative du progrès social et, pour ce faire conformément à la doctrine de l'Eglise, ils ont par ailleurs le devoir d'être dociles aux directives de l'Episcopat, lequel est le seul qualifié pour formuler les interprétations et juger les applications des principes. Mgr Roy rendit hommage au rôle dynamique des patrons dans la vie économique.

La deuxième journée du congrès fut consacrée à la tenue de la réunion générale annuelle de l'A.P.I. Enfin, un discours de M. Maurice Duplessis a clôturé le congrès. Le premier ministre a fait quelques déclarations sur des problèmes tels que l'entreprise privée, la centralisation, les grèves, le respect de l'autorité, etc.

Voici le texte des voeux adoptés par l'A.P.I.:

\section{Les grèves}

Attendu que le bien commun exige que l'ordre et et la paix soient maintenus dans la société;

Attendu que les ouvriers d'une entreprise ne peuvent être privés de leur droit au travail que pour des raisons graves et du consentement de la majorité;

Le congrès de l'Association professionnelle des industriels demande avec instance aux gouvernements de la province de Québec et du Canada d'adopter la législation nécessaire afin de décréter, en tenant compte des autres principes d'une juste grève, qu'aucune grève ne puisse être déclarée ou effectuée sans que la majorité des travailleurs de l'entreprise concernée ne l'aient votée librement au scrutin secret, sous la surveillance d'un représentant de lEtat et sans que le ou les motifs de la grève n'aient été expliqués clairement et en détail aux travailleurs intéressés.

\section{La collaboration patronale-ouvrière}

Considérant que la doctrine sociale de l'Eglise demande la collaboration entre patrons et ouvriers;

Considérant que l'intérêt des patrons et des ouvriers exige cette collaboration;

Considérant que l'un des buts principaux pour lesquels l'A.P.I. a été fondée est précisément la collaboration entre patrons et ouvriers;

Considérant que l'expérience pratique des patrons démontre les précieux avantages de cette collaboration;

L'Association professionnelle des industriels, réunie en congrès à Québec:

a) exprime sa vive satisfaction du travail accompli jusqu'à maintenant par ses membres pour la collaboration patronale-ouvrière;

b) insiste fortement pour que s'accentue partout cette collaboration dans un véritable esprit de justice et de charité;

c) recommande particulièrement le comité de coopération comme moyen efficace de réaliser et d'intensifier cette collaboration;

d) demande aux patrons de prendre en considération l'épanouissement de la personnalité des travailleurs au niveau de leur compétence, de leur responsabilité et de leurs risques.

\section{Sécurité sociale}

L'Association professionnelle des industriels, réunie en congrès plénier de ses membres:

1. Recommande que les formes de sécurité sociale qui pourraient être mises en vigueur tiennent compte de la part normale que les employeurs, les employés et l'Etat doivent avoir dans ce domaine.

2. Demande aux gouvernements et aux autres intéressés de procéder avec toute la prudence nécessaire afin de ne rien entreprendre qui puisse conduire directement ou indirectement au collectivisme.

\section{Projet d'association}

En vue de concrétiser les liens de naturelle sympathie qui existent entre les patrons italiens et les patrons canadiens, et afin de resserrer les relations établies entre l'Ucid. et l'A.P.I., à l'occasion du congrès de l'Uniapac à Rome, il est proposé et résolu que l'on fonde une association patronale italo-canadienne qui aurait pour but de développer les relations sociales, de fournir les renseignements industriels et autres et d'établir une plus étroite collaboration entre nos deux pays.

Le conseil serait composé de cinq directeurs canadiens, de cinq directeurs italiens et d'un secrétaire.

\section{L'Uniapac}

L'Association professionnelle des industriels, réunie en congrès plénier à Québec, réitère son entière adhésion 
à l'Union internationale des associations patronales catholiques et redit son désir de collaborer avec les autres patrons chrétiens de l'univers pour la réalisation de la doctrine sociale chrétienne.

Elle adresse ses félicitations à l'Association des patrons catholiques d'Italie pour l'éclatant succès du congrès de Rome, en mai dernier.

Elle adhère entièrement aux directives du Saint-Père dans son allocution aux patrons de l'univers, que l'on considère comme le charte des patrons chrétiens.

STATISTIQUES ET INFORMATION

I-ARBITRAGES EN COURS AU 31 OCTOBRE 1949

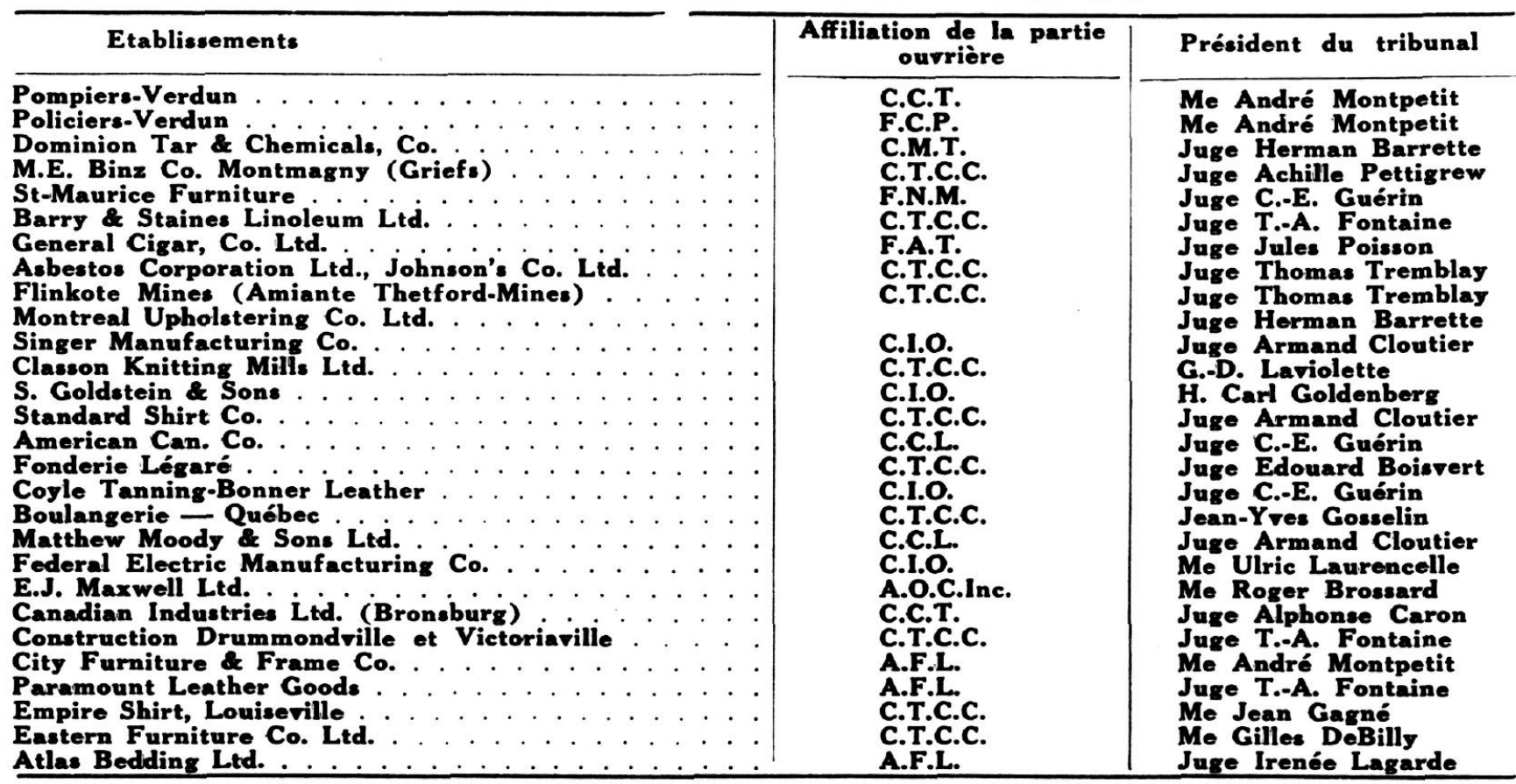

II-SENTENCES RENDUES ENTRE LE ler ET 31 OCTOBRE 1949

\begin{tabular}{|c|c|c|}
\hline Etablissements & $\begin{array}{l}\text { Affiliation de la } \\
\text { partie ouvrière }\end{array}$ & Date de la sentence \\
\hline 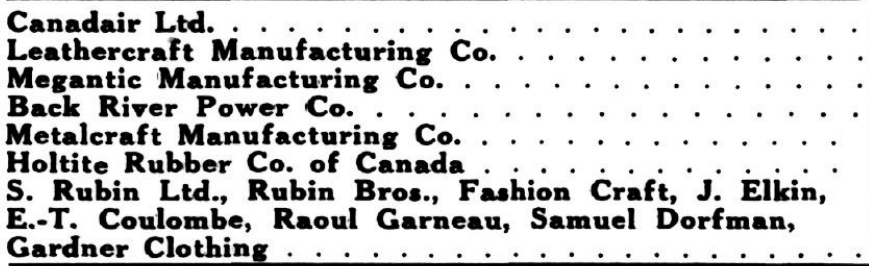 & $\begin{array}{l}\text { C.M.T.C. } \\
\text { F.A.T. } \\
\text { C.T.C.C. } \\
\text { C.T.C.C. } \\
\text { C.I.O. } \\
\text { C.T.C.C. } \\
\text { F.N.T.V. }\end{array}$ & $\begin{array}{r}3-10-49 \\
13-10-49 \\
7-10-49 \\
12-10-49 \\
13-10-49 \\
21-10-49 \\
24-10-49\end{array}$ \\
\hline
\end{tabular}

\section{JURISPRUDENCCE DU TRAVAIL}

Afin de renseigner les lecteurs du Bulletin, le Service de recherches entreprend une chronique mensuelle de Jurisprudence du travail. En principe, cette chronique portera sur les cas courants de jurisprudence soit des cours civiles, comme la Cour Supérieure ou la Cour du Banc du Roi, soit des tribunaux d'arbitrage ou encore sur les décisions intéressantes des diverses commissions administratives provinciales ou fédérales. Il pourra même arriver qu'on étudie des décisions intéressantes des cours étrangères. Bien que notre but soit surtout de nous attacher aux cas courants, il pourra arriver que cette chronique fasse un retour sur le passé afin de présenter aux lecteurs l'analyse de cas qui demeurent, malgré le temps, dune grande actualité. On n'est pas sans savoir, en effet, que la jurisprudence prend ses sources les plus fermes dans des décisions qui datent.

Effet du changement de personnalité juridique des parties à une convention

J.-L. Vachon \& Fils signe une convention collective avec les représentants de ses employés formés en association non incorporée. Après la signature, J.-L. Vachon \& Fils devient J.-L. Vachon \& Fils Ltée, par acte de vente, changeant totalement la personnalité juridique de l'employeur.

D'autre part, l'association non-incorporée devient le « Syndicat du bâtiment et bois ouvré de Beauce \$, incorporé selon la Loi des syndicats professionnels de Québec. Là encore, il y a changement de personnalité juridique. 